\title{
L'identification des relations de discours implicites: le cas de l'adversation
}

\author{
Gilles Corminboeuf \\ Fonds national de la recherche suisse (FNS) \& ATILF (CNRS - Université de Lorraine)
}

\section{Préalables}

\subsection{Marquage et non marquage des relations de discours}

Je parle de «non marquage » (Corminboeuf 2009) lorsqu'il y a absence de connecteur entre les membres d'une construction pour signifier une relation sémantico-pragmatique, qu'elle soit hypothétique, oppositive, causale ou temporelle. Dans (1) à (4), aucun connecteur adversatif du genre mais, pourtant, tandis que, or, etc. ne vient signifier la valeur adversative :

(1) Je connaissais beaucoup de fournisseurs, la plupart sont morts. (Rufin, Asmara et les causes perdues)

(2) Le texte de Meillet pose la question, il n’y répond pas. (discours scientifique)

(3) Les jeunes filles sont toutes faites pour des monstres, beaux ou hideux, et elles sont données à des hommes. De là leur vie gâchée. (Giraudoux, Judith, II-7)

(4) Il mange tous les jours au restaurant et il est maigre comme un fil. (cité par Riegel \& al.)

L'expression de l'adversation en français peut donc faire l'économie de «connecteurs oppositifs » ${ }^{1}$. Ces constructions sont ou du type asyndétique (juxtaposées) comme (1)-(2), ou du type syndétique (connectées par et) comme (3)-(4). Je considère que les cas de connexion par et du type (3) et (4) relèvent du non marquage de ces relations. Bien que la plasticité fonctionnelle du relateur et le rende compatible avec un grand nombre de relations ${ }^{2}$, il n'est pas spécialisé pour signifier l'opposition.

Les organisations argumentatives étudiées consistent en des assemblages d'unités prédicatives, qui affichent une posture argumentative anti-orientée - d'où cette interprétation adversative, qui est à reconstruire par inférence ${ }^{3}$. La valeur d'opposition est le résultat d'un pontage inférentiel (Clark 1977, Corminboeuf 2009) entre les membres d'une routine discursive (généralement binaire). Au plan formel, la construction est formée d'énonciations syntaxiquement indépendantes, mais qui forment ensemble une unité de sens.

\subsection{Contextualisation de la recherche}

Les constructions (1) à (4) rejoignent certains des objectifs d'une vaste recherche sur les constructions paratactiques (Corminboeuf 2009, 2010, 2013a, 2013b, à par.) :

- Démontrer qu'il n'y a pas de synonymie entre marquage et non marquage des relations de discours, du moins qu'il ne s'agit pas le même mode de donation du sens. Les constructions non marquées sont rarement réductibles à une paraphrase unique (au moyen d'un connecteur), voire à une paraphrase tout court. En conséquence, structures marquées et structures non marquées ne sont pas des variantes libres : l'option non marquée est le résultat d'un choix délibéré.

- Identifier les facteurs qui déterminent la sélection d'une construction non marquée.

- Saisir les fonctions du non marquage, à partir de l'entrée possible que constituent les constructions du type (1) à (4). Un rendement fondamental est celui d'indéterminer les relations de discours, mais 
il a aussi pour fonction d'impliciter des jugements de valeur ou des composantes « émotives », ce qui revient à différer leur prise en charge.

- Modéliser l'opposition entre juxtaposition (asyndèse) et connexion au moyen de et (syndèse), un aspect qui, à ma connaissance, est très peu documenté dans la littérature scientifique.

Suite à ces quelques remarques préalables qui délimitent l'objet d'étude et qui situent la recherche, je présenterai brièvement la relation de discours Contraste en SDRT telle qu'elle est appréhendée par Busquets $(\S 2$.). J'étudierai ensuite trois organisations argumentatives du français qui ressortissent à l'expression d'une relation adversative (§ 3. à §6.).

\section{2 «Contraste », une archi-relation de discours}

Selon Busquets (2007), à qui j'emprunte les exemples mentionnés dans ce paragraphe, la relation Contraste subsume plusieurs types d'oppositions :

- Les «Semantic oppositions ", par exemple John is quick but Bill is slow. Il y a un contraste (une dissemblance) entre deux états de choses qui partagent par ailleurs des similarités lexico-syntaxiques (une ressemblance). Les deux faits sont comparés, mais sans qu'ils soient en relation de contradiction.

- Les «Denials of expectation», par exemple John is a good student, but he's sloppy. Le second membre réfute une attente qui émerge du premier membre (il y a une relation de cause à effet sousjacente). On peut inférer du premier membre de la construction que les bons étudiants ne sont normalement pas confus ou négligés, ce qui s'oppose au contenu du second membre.

- Les enchaînements négatifs du type He does not have a car, but he has a bike où, si je saisis bien, le connecteur but endosse une valeur " rectificative », autrement dit il est du type sino / sondern (ou sonst) (Anscombre \& Ducrot 1977, Corminboeuf 2013a). Il s'agit d'une construction assez différente des deux premières classes de structures.

- $\quad$ Les relations Antithesis et Otherwise de Mann \& Thompson $(1985,1988)^{4}$.

Busquets (ibid., 105) précise par ailleurs que la relation de discours Contraste « occurs in dialogue also, as in the case of Corrections, Rejections, or Disagreements " (voir Spenader \& Maier 2009 pour un panorama des stratégies dénégatives).

Il est pour le moins ambitieux de regrouper tous ces cas de figure sous une seule relation de discours ${ }^{5}$. En limitant mon propos aux deux premières catégories, je serai moins audacieux que Busquets. Je ferai l'hypothèse que la seconde catégorie de Busquets - les «Denials of expectation » - regroupe deux organisations distinctes. J'étudierai donc trois organisations non marquées (i.e. implicites) à valeur adversative en français: les simples oppositions sémantiques (§ 3.), les organisations contreargumentatives «indirectes» comme les exemples (1)-(2) (§ 5.), et les organisations contreargumentatives « directes » comme les exemples (3)-(4) (§ 6.). Le § 4. établira la distinction entre contreargumentation directe et contre-argumentation indirecte.

\section{3 «Simples » oppositions sémantiques}

\subsection{Semantic opposition vs Denial of expectation}

La paternité de cette distinction entre « Semantic opposition » (5) et «Denial of expectation » (6) revient à Lakoff $(1971)^{6}$ :

(5) John is tall but Bill is short. (cité par Lakoff 1971)

(6) John is tall but he's no good at basketball. (ibid.) 
Les exemples (5) et (6) sont des constructions marquées, en l'occurrence par but. Dans (5), on observe une paire d'antonymes (tall vs short), mais pas de relation implicite évidente (par exemple une relation causale) entre les deux membres. Ainsi, ce n'est pas parce que John est grand que Bill est petit ${ }^{7}$.

Dans (6) en revanche, le locuteur établit un rapport entre 'être grand' et 'être bon au basketball'. Si quelqu'un est de grande taille, on attendra de lui qu'il soit un bon joueur de basketball : dans l'exemple mentionné, l'attente en question est déçue (John n'est pas un bon basketteur). Le recours à la notion de « topos $»^{8} \mathrm{~d}$ 'Anscombre et Ducrot est ici congru ( plus on est grand, plus on est susceptible d'être bon au basket »).

Pour les enchaînements en and, c'est-dire ceux qui sont "non marqués », Lakoff mentionne deux constructions qu'elle juge étranges mais, précise-t-elle, d'une étrangeté qui n'est cependant pas du même ordre :

(7) John has a Ph.D. in linguistics and he can read and write. (cité par Lakoff 1971)

(8) John has a Ph.D. in linguistics and he can’t read or write. (ibid., 125)

Dans (7), he can read and write est inférable de John has a Ph.D. in linguistics. Dire de quelqu'un qu'il a un doctorat en linguistique sous-entend qu'il sait lire et écrire. Il s'agit d'un sous-entendu très plausible, doté de beaucoup de crédit, autrement dit presque une inférence nécessaire. Lakoff parle d'ailleurs de « présupposé ». Dans la modélisation des phénomènes implicites dont je me sers - celle de Ducrot (1984) - il s'agit d'un sous-entendu, puisqu'il est annulable (ce que montre l'exemple (8)). L'information he can read and write est redondante parce que pour ainsi dire entraînée par le premier membre. D'où son étrangeté. Bien qu'il soit instructif quant au mécanisme inférentiel mis en jeu, on peut douter qu'un exemple du type (7) - sous-informatif et pléonastique (« étrange » pour Lakoff) - soit réellement bien représenté dans la communauté parlante9.

Dans (8), l'étrangeté présumée est de nature radicalement différente de celle de (7). Le second membre réfute contre toute attente cet implicite attaché au premier membre. A mon sens, (8) n'a rien d'une manœuvre rhétorique étrange : les exemples (3) et (4) sont aussi de ce type et le procédé est aisément attestable en français, aussi bien à l'oral qu'à l'écrit (voir § 6., infra). On notera que dans (8), on obtient en quelque sorte une plus-value implicite : un jugement dépréciatif est porté sur John (il est par exemple possiblement sous-entendu qu'il a plagié). On souligne une bizarrerie - être à la fois docteur et analphabète - ou du moins une situation insolite (Blakemore \& Carston 2005, Corminboeuf 2013b).

\subsection{Les « simples » oppositions sémantiques: matériel lexical spécifique mobilisé}

Dans les simples oppositions sémantiques, la relation de contraste résulte des parallélismes dans le lexique et dans la syntaxe :

(9) Les Allemands faisaient crever, les Français laissaient crever. (Petersen, Les oubliées)

(10) Chaque jour à vivre est une victoire, chaque jour vécu est une défaite. (attribué à $F$. Dard)

(11) Et voilà, soudain la vive lumière de dehors nous enveloppa; il faisait obscur, il fit clair ; il faisait froid, il fit de nouveau chaud; (Ramuz, Vie de Samuel Belet)

On notera les similarités dans la structure syntaxique, dans les tiroirs verbaux (pour (9)-(10)) et dans le lexique (les antonymes victoire vs défaite, obscur vs clair, froid vs chaud).

Selon Asher \& Lascarides (2003 : 168-169), le contraste dans les Semantic oppositions n'est en général pas exprimé par un connecteur. Le Bidois (1967 : 501) soulignait déjà que pour exprimer l'opposition, il suffit de rapprocher deux propositions - en contrastant des pronoms personnels et les signifiés des verbes -, sans avoir à les relier par une «ligature »spéciale : Vous avez parlé, j'ai agi. Autrement dit, les simples oppositions sémantiques sont par défaut des enchaînements non marqués (ce qui est plutôt 
exceptionnel). La finalité serait au contraire une relation toujours marquée en français (par afin de/que et pour).

Les constructions (9)-(11) sont asyndétiques, mais les enchaînements articulés par et, bien que rares, sont néanmoins attestés :

(12) il était toujours numéro un et j'étais toujours numéro deux + il avait toujours le prix d'excel- le prix d'- les félicitations et j'avais toujours le prix d'excellence + et pendant des années comme ça il a été premier et moi deuxième + jusqu'à ce que j'aille au collège (oral, pfc, Puteaux-Courbevoie, 99aaf1, gg) ${ }^{10}$

Là également, on observe des parallélismes remarquables, aussi bien au plan lexical qu'au niveau du choix des temps verbaux, une concomitance temporelle d'une énonciation à l'autre (soit une forme de ressemblance). Et une opposition sémantique (numéro un vs numéro deux; premier vs deuxième; il vs je...), soit une forme de dissemblance qui fonde le contraste.

Au total, dans les simples oppositions sémantiques, le contraste est construit essentiellement au moyen de parallélismes dans la structure lexicale et syntaxique. L'absence (par défaut) de connecteur entre les membres de la construction s'explique par le fait que les connecteurs sont les véhicules de topoï. Or une des caractéristiques des simples oppositions sémantiques est l'absence de topos activé entre les deux membres de la routine (voir note 7).

Je vais maintenant laisser de côté les simples oppositions sémantiques pour distinguer deux classes de faits à l'intérieur des cas de « dénis d'attente » de Lakoff.

\section{Contre-argumentation directe vs contre-argumentation indirecte}

Le travail d'Anscombre (2002) constitue un bon point d'ancrage pour décrire la différence entre deux grands types de routines adversatives illustrés par (1)-(2) vs (3)-(4). Anscombre étudie des enchaînements adversatifs de type $\{\mathrm{p}$ mais / pourtant $\mathrm{q}\}$, c'est-à-dire marqués au moyen des connecteurs mais ou pourtant $^{11}$.

L'auteur distingue la contre-argumentation « directe » et la contre-argumentation « indirecte ». Dans l'opposition directe, $\mathrm{p}$ est un argument pour non-q. C'est le cas des enchaînements du type pourtant ${ }^{12}$ comme (13) et (14) :

(13) Les autruches sont des oiseaux, mais / pourtant elles ne volent pas. (cité par Anscombre $2002: 123)$

(14) Ils fuyaient le voisinage et redoutaient pourtant la solitude. (ibid., 120)

Dans (14), « fuir le voisinage » (le membre p) est un argument pour « redouter la compagnie » - on fuit le voisinage parce qu'on redoute la compagnie -, qui s'oppose directement à « redouter la solitude » (le terme q). La connexion se fait entre q et un contenu qui implique non-q. On notera dans ce même exemple l'association de et avec pourtant.

Dans la contre-argumentation indirecte, $\mathrm{q}$ est un argument pour une conclusion $\mathrm{r}$ et $\mathrm{p}$ est argument pour non-r :

(15) Je ne prends pas de dessert : j’adore le sucré, mais ça fait grossir. (ibid.)

Dans (15), le segment ça fait grossir (q) est un argument pour $\mathrm{r}<\mathrm{je}$ ne mangerai pas de dessert $>$ et j'adore le sucré (p) est un argument pour non-r $<$ je mangerai du dessert $>$. L'opposition entre les deux conclusions argumentatives anti-orientées est dite indirecte.

Pour Anscombre, l'exception que présente mais est compatible avec le topos; il s'agirait d'une « exception ordinaire» qui renforce le topos. En revanche, pourtant présenterait une exception qui disqualifie le topos convoqué (une « exception extraordinaire », dans les termes d'Anscombre). Au total, 
avec mais on exploiterait le topos, la règle serait confirmée, alors qu'avec pourtant on révoquerait le topos, la règle serait infirmée.

La description d'Anscombre me semble peu ou prou transposable (avec les précautions d'usage) aux enchaînements «non marqués ». En m'inspirant de l'opposition binaire d'Anscombre, je vais distinguer deux types d'assemblages adversatifs non marqués (en plus des simples oppositions sémantiques), les contre-argumentations indirectes et les contre-argumentations directes.

L’enchaînement non marqué (16) est indirect :

(16) L1 : puis les garçons ils ont pas envie de quitter le nid tu vois qu'ils sont très bien:

L2 : [c'est vrai ?]

L2 : ah oui ? pourtant ils gagnent leur vie: ?

L1 : [oui] oui ben peut-être que-

L2 : ils doivent bien avoir des petites copines euh: ? ça c'est-

L1 : David en a eu une c'est cassé + et Guillaume je crois qu'il y a quelque chose avec $(\mathrm{XXX})+$ je sais pas en ce moment elle est partie je sais pas où traîner là + au Burkina Faso je crois qu'elle est (oral, pfc, Vendée, 85amm1, lg)

« Avoir une copine » (David en a eu une) est un argument pour « quitter le nid », alors que « c'est cassé » (le membre q) est un argument pour ne pas le quitter; l'opposition est indirecte, au sens d'Anscombre. On comprend la construction plutôt au sens de «David a eu une copine, mais c'est cassé » qu'au sens de «? David a eu une copine, pourtant c'est cassé ${ }^{13}$.

Le slogan politique pour le moins surréaliste guillemeté en (17) est en revanche un enchaînement direct :

(17) Chef de l'une des factions qui se disputaient le pouvoir au Liberia à partir de 1989, il encouragea alors ses troupes d'enfants soldats à se livrer à une surenchère macabre : viols, pillages, mutilations et même cannibalisme, qui transformèrent le pays en enfer. Jusqu'à ce qu'il soit élu... président du pays en 1997, recueillant 75\% des voix avec ce slogan : « Il a tué ma mère, il a tué mon père et je vote pour lui ». En réalité, anéantis par dix ans d'inhumanité, les Libériens savaient qu'ils n'avaient pas d'autre choix que de le laisser accéder au pouvoir en échange de la paix. (presse écrite)

Les deux énonciations frontales sont un argument possible pour «je ne voterai pas pour lui » (en général on n'est pas porté à soutenir l'assassin de sa famille), qui s'oppose directement à je vote pour lui. On paraphraserait plus volontiers la construction au moyen de pourtant que de mais.

Mais l'un des défis de cette recherche est de comprendre cette distinction argumentative sans le recours à la paraphrase. C'est pourquoi je vais exploiter des critères formels permettant de distinguer les constructions du type (1)-(2)-(16) de celles du type (3)-(4)-(8)-(17). Pour chaque type d'enchaînement, il $\mathrm{y}$ a récurrence d'un assemblage d'indices morpho-syntaxiques.

\section{Les enchaînements indirects : ressorts de la valeur adversative}

\subsection{Oppositions modales, aspectuelles, lexicales}

Comme pour les simples oppositions sémantiques, la nature argumentative oppositive des deux membres de la construction est inscrite dans la structure. Un grand nombre d'exemples comporte en effet une opposition de modalité, avec communément une négation dans un des deux termes, généralement dans le second :

(18) L1 : et y avait tellement de monde en fait quand on est arrivé + il restait plus que des places au premier rang

L2 : (XXX) 
L1 : c'était au César + et en fait euh: + moi j'avais déjà vu des films au premier rang ça m'avait pas gêné + mais là je sais pas si c'est parce que la salle est pas si grande et- + 1- le premier rang il est vraiment Collé à l'écran + ah putain: + ah putain on n'en POUvait plus quoi (oral, cid_cm ; à propos d'une séance de cinéma)

(19) Ingrid est obligée d'aller à Stockholm pour une affaire urgente. Elle me propose de l'accompagner, je ne veux pas. Elle me propose que quelqu'un vienne me tenir compagnie pendant les journées où elle sera absente, je le veux encore moins. (Bergman, Laterna magica)

Une opposition aspectuelle ou temporelle peut être pareillement au service de l'effet de contraste - bien qu'il y ait très souvent le même tiroir verbal dans les deux énonciations :

(20) L1 : ils avaient- ils avaient une:- ils avaient une petite usine à Cholet

L2 : ouais

L1 : ils avaient commencé à Cholet ils se sont décentralisés + ils avaient gardé leur usine à Cholet mais ils en ont créé une autre là + et puis après là ça a été le le siège social

L2 : d'accord + et donc ça a commencé chez votre grand-mère ?

L1 : oui ça a commencé chez ma grand-mère oui (oral, pfc, Vendée, 85asl1, lg)

Dans (20), le plus-que-parfait du premier membre (avaient commencé) s'oppose au passé composé du second membre (se sont décentralisés).

La structure rythmique et métrique du titre (21) est remarquable. Il s'agit d'un alexandrin avec une paronomase (se poursuivent $-s$ 'amenuisent) et une charpente formelle identique $(\mathrm{SN}+s e-\mathrm{V})$ :

(21) Steve Fossett : les recherches se poursuivent, les espoirs s'amenuisent. (presse écrite, titre)

Une lecture possible identifierait une opposition argumentative entre les recherches se poursuivent qui argumente en faveur de $<$ il y a encore de l'espoir $>$ et les espoirs $s$ 'amenuisent qui oriente en faveur de $<$ il n'y a plus guère d'espoir> (plus les recherches durent, moins il y a d'espoir). Il y a à la fois contraste argumentatif et procédés de cohésion au sein de la routine : en effet, les deux énonciations sont opposées au plan argumentatif (dissemblance), mais elles forment une unité périodique solidaire (ressemblance). Ces routines constituent des "périodes », au sens que le XVIIème siècle donnait à ce terme, c'est-à-dire des structures marquées par des parallélismes et des oppositions.

\subsection{Asymétrie de la construction}

J'entends par «(a)symétrie» la permutation possible ou non des membres de la construction. Les enchaînements indirects sont généralement non réversibles, à témoin les exemples (1), (2), (16), (22) et (23) :

(22) Et brusquement elle reprit : «A la prochaine fois. » Je voulus la retenir, elle était déjà loin. Je l'appelai par son nom, l'écho seul me répondit. (Ramuz, Vie de Samuel Belet)

(23) et en plus on est arrivé un jour de grève + et justement à la gare on a dit écoutez on part euh à Naples est-ce qu'on va arriver? + ouh: ben alors il nous a dit vous êtes partis vous êtes pas sûrs d'arriver + oh bonté divine alors là l'angoisse (oral, pfc, Roanne, 42acd1, lg)

Ce sont des contraintes d'ordre liées au sémantisme des prédicats verbaux et à la chronologie événementielle qui prévalent. Ainsi, dans (22), on ne peut pas retenir quelqu'un qui est déjà loin ou entendre la réponse de l'écho avant d'avoir appelé. Le fragment (23) active un schéma d'actions ordonnées (on part avant d'arriver). Deux verbes sémantiquement antithétiques (partir vs arriver) sont introduits de la même manière (vous êtes...), ce qui renforce le parallélisme du tour. La logique de succession des actions communicatives rend peu praticable une permutation des énonciations. 


\subsection{Préférence pour les enchaînements asyndétiques}

Les enchaînements indirects sont généralement juxtaposés. Parfois, comme dans (23), une connexion au moyen de et serait difficile à réaliser, à conditions sémantiques similaires (voir également l'exemple $20)^{14}$ :

(23') "?vous êtes partis et vous êtes pas sûrs d'arriver

Cela a probablement un rapport avec le fait que la construction est asymétrique et, du coup, ses membres non permutables (§ 5.2.) :

(23") "?vous êtes pas sûrs d'arriver vous êtes partis

$\mathrm{Au}$ total, les enchaînements indirects sont des routines qui s'appuient communément sur un topos contrairement aux simples oppositions sémantiques. Ainsi dans (18), le topos convoqué serait : «plus on est proche de l'écran, moins l'emplacement est agréable ». On observe par ailleurs que le contraste est marqué dans la morpho-syntaxe (au moyen d'oppositions modales, de verbes à signifiés proches ou appartenant à même classe sémantique). Enfin, l'asymétrie de ces enchaînements est solidaire du fait qu'ils sont préférentiellement juxtaposés (et non connectés par $e t$ ).

\section{Les enchaînements directs : matrice d'indices au service de l'attente déçue}

Je vais maintenant étudier les enchainements directs du type (3)-(4)-(8)-(17). Ils sont moins courants que les enchaînements indirects, mais plus intéressants pour cerner le rendement fonctionnel du connecteur $e t$, puisqu'ils sont pour ainsi dire systématiquement articulés par $e t$.

\subsection{Effet d'attente trompée}

Contrairement à Lakoff (1971), je réserve l'effet d'attente trompée pour les enchaînements directs. Je définirai une " attente » pragmatique comme une prévision à caractère plausible, qui se fait par abduction. Elle prend la forme d'un sous-entendu dont le crédit est très élevé, ce que montrait assez bien l'exemple (8). La force d'une attente est fonction du degré de confiance qu'on lui accorde.

Dans les enchaînements directs, la seconde énonciation enchaîne en général sur un sous-entendu doté de beaucoup de crédit, inférable de la première énonciation pour en réfuter la conséquence. Voyons un exemple, où il est question de la méchanceté d'une institutrice :

(24) elle nous criait toujours dessus (...) et vient le jour où l'inspecteur est passé et elle est devenue toute mielleuse toute gentille (...) j'ai sorti une phrase bourrée de fautes de grammaire d'orthographe de tout ce que on peut imaginer et elle a été très gentille + mais reprenez Lucie euh euh continuez enfin on allait pouvoir euh (rires) et euh le la semaine suivante il y avait pas l'inspecteur + elle était redevenue euh aussi méchante qu'avant (rires) (oral, pfc, Toulouse, $31 \mathrm{afgc} 1, \lg$; à propos d'une institutrice)

On observe des quantifieurs (bourrée de, tout ce qu'on peut imaginer) qui contribuent à élaborer le sousentendu que la performance de l'élève pourrait justifier une sanction. Or, "contre toute attente», l'institutrice a été très gentille. Le sous-entendu élaboré dans le premier membre se voit doté d'un degré de confiance élevé, et le second membre vient in fine déjouer cette attente forte. On peut penser ici au caractère annulable des sous-entendus : on en joue, en exploitant des schémas d'action routinisés, en révoquant des topoï. Dans (24), le contenu de q s'oppose directement à l'implicite qu'on tire de p.

Au plan argumentatif, un (ou plusieurs arguments) en faveur d'un sous-entendu est, contre toute attente, invalidé dans le second membre. On peut postuler que le rendement argumentatif principal de ces constructions $\{\mathrm{p}$ et $\mathrm{q}\}$ est la « révision d'une attente forte». 
Un mot sur la « surprise » dont il est souvent question (Blakemore \& Carston $2005: 581$ ), un effet qui est une conséquence de ce mécanisme d'attente trompée. Elle est, d'une part, généralement anticipée par des informations contextuelles: la valeur adversative est en quelque sorte préfabriquée (il s'agit d'une surprise préparée). D'autre part, comme la manœuvre argumentative est codifiée, on fait mine de ménager un effet de surprise : or, évidemment, personne n'est dupe (Corminboeuf 2010). Voyons (25) qui illustre bien le fait que la relation est souvent pré-conçue dans l'avant-discours :

(25) L1 : non mais c'est vrai parce que cette fille-là elle a vraiment un moral euh + enfin elle relle rigole tout le temps quoi +

L2 : $\quad[\mathrm{mmh}]$

L1 : elle était sur son lit d'hôpital + elle a m-même pas encore de corset ni rien + elle avait juste des antidouleurs et elle rigolait malade enfin c'était: (oral, pfc, Liège, blasl1, lg)

Les énonciations elle était sur son lit d'hôpital, elle a même pas encore de corset ni rien et elle avait juste des anti-douleurs contribuent toutes trois à construire le sous-entendu que la situation ne prêtait pas à rigoler. Mais on s'attend à un retournement argumentatif, en raison de l'assertion préalable cette fille-là a vraiment un moral elle rigole tout le temps. En fait, la construction en italique vient étayer cette assertion.

\subsection{Indices morpho-syntaxiques liés au phénomène d'attente contrariée}

6.2.1. Au plan morpho-syntaxique, on observe souvent des marqueurs scalaires, - je les ai signalés pour (24) - i.e. des indices qui signifient de petites ou de grandes quantités sur une échelle de valeurs. Dans (4) par exemple, l'opposition se fait entre le quantificateur tous et l'hyperbole maigre comme un fil. Ces marqueurs sont soulignés en italiques dans (26) et (27) :

(26) L1 : donc ils sont là pour nous former + pour nous faire rentrer euh: un certain nombre de choses dans la tête

L2 : [et les profs c'est surtout des hommes ?]

L1 : pour nous imposer une rigueur + oui oui alors moi je réfléchis + j'avais une majorité d'hommes + j'avais quelques femmes et tu vois et c'est les femmes qui m'ont posé le plus de soucis + c'étaient les plus vaches quoi (oral, pfc, $75 \mathrm{xcm} 1$, gg; à propos des profs)

(27) Il venait de s'apercevoir d'une chose inouïe : les bracelets d'or n'étaient que de la vulgaire camelote. (...) Ces bracelets valaient peut-être quelques piastres, et il était allé jusqu'au meurtre pour se les approprier. (Cossery, Mendiants et orgueilleux)

Dans (26), on notera le superlatif (le plus de soucis, les plus vaches) qui s'oppose à un quelques (vs une majorité) à valeur de quantité faible. Dans (27), allé jusqu'au meurtre - qui constitue la plus grande extrémité - est mis en opposition avec quelques piastres, c'est-à-dire une somme dérisoire.

On peut faire l'hypothèse que ces indices de haut degré sont au service de la création de l'effet d'attente forte contrariée. Il existe une corrélation entre cette attente déjouée et les indices scalaires propres aux enchaînements directs (ceci contrairement à ce qu'on observe dans les enchaînements indirects).

6.2.2. D'autres marqueurs sont bien représentés dans les enchaînements directs. Les lexies comme en fait, en réalité, ou en fin de compte signalent une réévaluation entre le contenu du premier membre et celui du second membre de la construction. Ces connecteurs attestent en quelque sorte que la contradiction est assumée par le locuteur. Dans (28), il est question d'une jeune journaliste à qui on conseille d'insérer dans ses papiers des indications qui suggèrent qu'elle est allée « sur le terrain » :

(28) quand je partais en reportage on me disait mais + dis un truc + qui euh qui montre que tu y as été + donne un détail vivant qui qui montre que tu y es allée par exemple je sais pas euh + est-ce que il y avait la tempête enfin tu vois c'est des conneries mais + tu vois bon et 
souvent le mec qui me- un des un des mecs qui me reprenait- par exemple j'étais allée à Verdun un jour + et on sentait pas en fait que j'y étais allée (oral, crfp, pro-pso-1)

(29) moi je regarde la télé euh: le midi aux informations où on dit ah il faut tout faire pour aider le petit commerce + et en fin de compte on fait tout pour nuire au petit commerce + et on avantage euh beaucoup les grandes surfaces euh: (oral, crfp, pro-qui-1)

Charolles rend compte comme suit de la fonction d'en réalité et d'en fin de compte :

\begin{abstract}
en réalité et en fin de compte signalent (...) que celui qui les énonce a trouvé un contexte dans lequel l'incompatibilité n'a plus cours (à charge pour le récepteur interprétant de 'remplir' ce contexte qui est présenté comme étant au-delà des apparences - en réalité - ou comme postérieur à un développement événementiel - en fin de compte). (Charolles $1984: 102$ )
\end{abstract}

L'auteur ajoute que ces connecteurs ont «pour fonction d'articuler des contenus opposés de telle sorte que les énoncés dans lesquels ils figurent ne paraissent pas contradictoires ». D'autres locutions prépositionnelles peuvent remplir un rôle apparenté :

(30) Nous étouffons sous un trop-plein d'informations et, en même temps, les messages essentiels, capables de transformer notre vie, n'atteignent plus notre conscience. (Tarkovski, Le temps scellé)

Les indices scalaires et les connecteurs comme en fait ou en réalité, témoignent d'une forme de routinisation de la structure. Les indices scalaires sont les auxiliaires de l'effet d'attente trompée et les connecteurs stimulent la résolution de ce qui n'est qu'en apparence une contradiction.

Le choix du connecteur et (et non d'un connecteur adversatif comme pourtant ou mais) remplit aussi la fonction de signaler que les deux conjoints ne sont pas si antithétiques qu'on pourrait le penser.

\title{
6.3 Symétrie (apparente) de la construction
}

Un autre moyen de différencier les deux types d'enchaînements est de juger du caractère symétrique (= permutable, réversible) ou non des membres de la construction. Comme les simples oppositions sémantiques, les enchaînements directs tolèrent a priori mieux la réversibilité des membres que les enchaînements indirects. L'exemple (31) met en lumière ce principe de symétrisation :

(31) Tu me traites comme moins que rien, et tu me demandes de t'aider. (cité par Anscombre $2002: 125-126)$

Tu me demandes de t'aider, et tu me traites comme moins que rien. (ibid.)

L'argumentation circulaire du dialogue (32), illustre aussi l'apparente symétrie des enchaînements directs :

(32) L1 : j'ai mes mes collègues qui sont dans des studios + donc c'est trouvé dans un foyer logun foyer trouvé par le: les impôts

L2 : $\quad$ [par les impôts ?]

L1 : $\quad$ eh ben elles payent nettement moins cher

L2: $\quad$ [ah bon]

L1 : $\quad$ et elles ont plus de superficie $+c$ 'est

carrément des studios hein

L2 : et elles payent ah ouais moins cher (oral, pfc, Aveyronnais à Paris, 75xpd1, lg)

(32) présente la structure [p et q et p'], organisée sur deux tours de parole. A la fin de l'extrait, le locuteur L2 enchaîne au moyen de et $p$ ' sur la construction $p$ et $q$ de la locutrice L1.

Faut-il en conclure que et a une fonction de symétriseur? A mon sens, il s'agit plutôt d'une symétrie «de façade ». En effet, parler d'un mécanisme d'attente déçue (supra § 6.1.) suppose une forme d'iconicité dans la routine discursive : on ouvre une attente et ensuite on la déçoit (et non l'inverse). Une telle 
manœuvre rhétorique exclut la permutation des membres de la routine (voir Corminboeuf, à paraître, pour le détail de l'analyse) ${ }^{15}$.

\subsection{Caractère commun de la syndèse par et}

Les enchaînements directs sont généralement connectés par et, à tel point que la solution asyndétique est parfois inenvisageable. De Cornulier souligne par exemple que le retrait de et serait malvenu dans (33) :

(33) Ça n'a même pas son agrégation de grammaire et ça ose me tutoyer. (cité par de Cornulier $1985: 41)$

Dans (31) supra, on peut également difficilement faire l'économie de et. La juxtaposition est sans doute tenue pour non optimale par les sujets parlants qui, de fait, ne la pratiquent qu'exceptionnellement. En effet, les enchaînements directs asyndétiques sont extrêmement rares, et pour ceux-ci l'insertion d'un et ne poserait aucun problème. C'est le cas du vers de Racine (34) et du mot de Godard (35) :

(34) Je l'aime, je le fuis ; Titus m'aime, il me quitte. (Racine, Bérénice, V, 7)

(35) Delon est un truand, il a une parole. Depardieu est un honnête homme, il n'en a pas. (presse écrite, attribué à J.-L. Godard)

Dans (34), le choix de l'asyndèse est peut-être guidé par des contraintes métriques. Dans (35), l'interprétation dépend du topos mobilisé, i.e. de l'image que l'on se fait d'un truand. Si on le conçoit comme quelqu'un qui n'a pas de parole, la relation est adversative. Si on le conçoit au contraire comme quelqu'un qui a une parole, la relation est résultative (conséquentielle). Godard joue ici argumentativement sur les deux tableaux. Si on veut lever l'ambiguïté et viser la lecture adversative, il faut fatalement mettre et (Delon a une parole et c'est un truand // Delon est un truand et il a une parole) : ainsi, on ne peut pas comprendre «donc c'est un truand » ou " donc il a une parole ${ }^{16}$. Le non marquage a cette propriété fondamentale d'indéterminer les relations de discours. Il semble donc que l'absence exceptionnelle $^{17}$ de et puisse le plus souvent s'expliquer - par exemple par des impératifs d'ordre métrique ou pour entretenir délibérément une ambiguïté.

Au total, dans les enchaînements contre-argumentatifs directs une attente forte est déçue au moyen de l'invalidation d'un topos, des indices morpho-syntaxiques atténuent la contradiction et la construction faussement symétrique - est presque systématiquement connectée par et.

Les organisations discursives directes et indirectes sont des routines duelles d'actions qui argumentent dans deux directions opposées. Le connecteur et est à placer du côté des enchaînements directs, alors que la solution juxtaposée est préférée dans les enchaînements indirects.

\section{Conclusions}

\subsection{Synthèse des propriétés des organisations contrastives étudiées}

Ci-dessous, le tableau résume les propriétés de chaque type d'organisation argumentative décrite : les simples oppositions sémantiques, les enchaînements contre-argumentatifs indirects et les enchaînements contre-argumentatifs directs.

\begin{tabular}{|l|l|l|l|l|}
\hline & (A)syndèse & (A)symétrie & $\begin{array}{l}\text { Appui ou non } \\
\text { sur un topos }\end{array}$ & $\begin{array}{l}\text { Indices } \\
\text { morphosyntaxiques }\end{array}$ \\
\hline $\begin{array}{l}\text { Simples oppositions } \\
\text { sémantiques } \\
\begin{array}{l}\text { Ex. : Pierre parle le français, } \\
\text { Marie parle l'anglais }\end{array}\end{array}$ & [+ asyndèse] & [+ symétrie] & $\begin{array}{l}\text { ne s'appuie pas } \\
\text { sur un topos }\end{array}$ & $\begin{array}{l}\text { forts parallélismes } \\
\text { lexico-syntaxiques }\end{array}$ \\
\hline
\end{tabular}




\begin{tabular}{|l|l|l|l|l|}
\hline $\begin{array}{l}\text { Enchaînements indirects } \\
\begin{array}{l}\text { Ex. : Le texte pose la } \\
\text { question, il n'y répond pas }\end{array}\end{array}$ & [+ asyndèse] & [-symétrie] & exploite un topos & $\begin{array}{l}\text { oppositions modales, } \\
\text { prédicats verbaux } \\
\text { apparentés }\end{array}$ \\
\hline $\begin{array}{l}\text { Enchaînements directs } \\
\begin{array}{l}\text { Ex. : Il mange tous les jours } \\
\text { au restaurant et il est maigre } \\
\text { comme un fil }\end{array}\end{array}$ & [- asyndèse] & [+/-symétrie ?] & révoque un topos & $\begin{array}{l}\text { indices scalaires, } \\
\text { connecteurs du type } \\
\text { en fait, en réalité }\end{array}$ \\
\hline
\end{tabular}

Figure 1: Tableau récapitulatif

\subsection{Pour une étude des relations de discours non marquées}

Les comptages effectués dans des corpus écrits en anglais, en espagnol et en français montrent d'ordinaire que de $50 \%$ à $75 \%$ des relations de discours sont non marquées (i.e. implicites), quelles que soient les options théoriques et méthodologiques adoptées : par exemple la SDRT et leur corpus " Annodis », les travaux s'inscrivant dans le paradigme de la Rhetorical Structure Theory de Mann \& Thompson (1988), les travaux en linguistique computationnelle comme celui de Marcu \& Echihabi (2002). Considérons les quatre citations significatives ci-dessous :

Finally, one of the most obvious problems with all the studies (including this one) on automatically identifying discourse relations is that they only work with marked discourse relations. Our results won't help much in identifying unmarked Contrast relations, yet these relations are very frequent. Carlson \& al. (2003) have shown that in the corpus of Rhetorical Structure trees only 61 out of 238 contrast relations were marked by a discourse marker. This means that contrastive markers would help to identify only $25 \%$ of contrast relations in that corpus. (...) Finding a solution to these problems will be a challenge for future work. (Spenader \& Lobanova 2009 : 10)

Few studies have considered all possible signals for discourse relations, and thus the perception is that signalling is low. Studies that consider only discourse markers usually show that over $50 \%$ of the relations are unsignalled. The analyses on the RST website (Mann \& Taboada, 2007), a very diverse collection comprising 187 units, have about $72 \%$ of the relations unsignalled (by a discourse marker). In a study of two different corpora, I found that relations were signalled about $31 \%$ of the time in conversation, and $44 \%$ in newspaper articles (Taboada, 2006), again with reference mostly to discourse markers, although a few other signals are discussed in that paper (mood, finiteness and punctuation). Many other signalling mechanisms remain understudied. (Taboada $2009: 128$ )

Previous reports of relation signalling, including my own work, have given figures of up to $70 \%$ of relations without signals. I have already pointed out that most of this research considered only discourse markers. In fact, some lines of research study coherence relations only if they are signalled by a marker. (...) For my book (Taboada, 2004), I analyzed 30 conversations in English and 30 in Spanish, annotating them with rhetorical relations. (...) The summary is that relations were marked $30.86 \%$ of the time in English, and $45.88 \%$ in Spanish. (Taboada $2009: 134$ )

It is worth noting that, with some exceptions, the study of how relations are signaled has mostly been confined to explicit discourse markers, preferably in written texts. Very little attention has been paid to other linguistic signals, including mood, modality, or intonation. (...) Future work should explore how readers construct representations for relations that are not explicitly signaled. (Taboada 2006 : 573; 589)

Ces quatre citations sont révélatrices de plusieurs éléments fondamentaux : 
- L'écrasante majorité des relations de discours en langue naturelle sont non marquées. Citons encore, pour la relation Contraste, Marcu \& Echihabi (2002) qui n'ont identifié - dans un corpus écrit en anglais - que $26 \%$ de relations contrastives marquées (le pourcentage est le même pour la relation Explanation-Evidence).

- Les recherches ne portent pour ainsi dire que sur les relations marquées, ce qui empêche de saisir globalement le fonctionnement du maillage rhétorique des discours. Les indices linguistiques qui en dehors des «marqueurs de discours »- signalent les relations de discours sont très peu étudiés dans les contributions sur les relations de discours.

- Les études portent presque exclusivement sur l'écrit.

Ce constat de l'approche très réductrice qui prévaut actuellement constitue un défi pour les recherches futures.

De surcroît, le centrage de l'analyse sur les connecteurs comporte d'autres inconvénients que celui de passer à côté de la grande majorité des relations de discours - qui sont donc non marquées.

- $\quad$ Si on dit qu'un connecteur marque une relation de discours, on introduit une circularité gênante entre la relation sémantique et le marquage morphologique (éventuel) de cette dernière. Taboada (2009) distingue opportunément l'étude des connecteurs et l'étude des relations rhétoriques, alors que l'on a trop tendance à les assimiler ${ }^{18}$.

- Il est fait abstraction de tous les autres indices, qui sont en quelque sorte incorporés dans la charge instructionnelle du connecteur. On reporte en effet sur celui-ci uniquement, une relation qui est en en réalité également construite par d'autres indices (temps verbaux, formes verbales non finies, modalités, signifiés des verbes, parallélismes structuraux, implicatures, etc.). Si on lui fait porter la responsabilité de marquer les relations, il se voit chargé d'instructions (Nølke 2001) qui lui sont partiellement étrangères. En reportant sur l'indice supposé massif (le connecteur) les composants du sens fournis par des indices plus subtils, on assigne à un niveau local (à tel marqueur de discours) le résultat d'un processus interprétatif qui est toujours global. En ce sens, un retour à Mann \& Thompson (1988) serait profitable.

- On fait comme si les connecteurs n'étaient pas polyfonctionnels. Pour Taboada (2006: 578), so est par exemple l'auxiliaire de huit relations et and de cinq relations de discours distinctes ${ }^{19}$. Il serait plus prudent de convenir qu'un connecteur comme et ne signale pas une relation de discours, mais qu'il en exclut tout au plus certaines (ce qui est différent), notamment la relation Explication.

- L'idée de vouloir à tout prix identifier une relation de discours entre chaque segment fait peu de cas de l'indétermination propre à tout discours en langue naturelle et dont les sujets parlants s'accommodent parfaitement (Corminboeuf 2010). Paradoxalement, une structure se voit surdéterminée, alors que - parce qu'elle est non marquée - elle a peut-être la finalité inverse, celle précisément de rester sous-déterminée.

Si la recherche en linguistique ne porte que sur des faits langagiers extractibles de manière automatique (en particulier les «marqueurs de discours »), on se prive du fonctionnement d'une bonne partie du système. Un « grand » corpus doit également servir à repérer les faits rares qui révèlent des choses sur les faits les plus courants, les données non intuitives et - dans le cas des relations de discours - à repérer et quantifier les faits communs mais non marqués. L'analyse outillée des grands corpus a l'avantage de permettre des facilités au plan de l'analyse quantitative, mais le défi (pas seulement pour la linguistique computationnelle) est maintenant de prendre en compte les phénomènes plus difficiles à extraire, par exemple les relations non marquées. Afin de pouvoir un jour rechercher automatiquement les relations de discours non marquées, il faut au préalable réaliser une description qualitative fine de ces relations implicites, entreprise à laquelle l'étude que je conclus ici apporte sa contribution.

\section{Références bibliographiques}

Anscombre, J-C. \& Ducrot O. (1977). Deux mais en français ? Lingua, 43, 23-40. 
Anscombre, J.-C. (2002). Mais / pourtant dans la contre-argumentation directe : raisonnement, généricité, et lexique. Linx, 46, 115-132.

Antoine, G. (2001=1958). La coordination en français. 2 vol., Paris : Ed. d'Artrey.

Asher, N. \& Lascarides A. (2003). Logics of conversation. Cambridge : CUP.

Blakemore D. \& Carston, R. (2005). The pragmatics of sentential conjunction with and. Lingua, 115-4, 569-589.

Busquets, J. (2007). Discourse Contrast : Types an Tokens. In Aurnague, M., Korta K. \& Larrazabal J. M. (eds), Language, Representation and Reasoning. Memorial Volume to Isabel Gómez Txurruka, Bilbao: University of Basque Country Press, 103-123.

Carel M. \& Ducrot O. (1999). Le problème du paradoxe dans une sémantique argumentative. Langue française, 123, $6-26$.

Carlson L., Marcu D. \& Okurowski M. E. (2003). Building a Discourse-Tagged Corpus in the Framework of Rhetorical Structure Theory. In van Kuppevelt J. \& Smith R. (eds), Current Directions in Discourse and Dialogue, Kluwer Academic Publishers, 85-112,

Charolles, M. (1984). En réalité et en fin de compte et la résolution des oppositions. Travaux du centre de recherches sémiologiques, 47, 81-111.

Clark, H. H. (1977). Bridging. In P. N. Johnson-Laird \& P.C. Wason (éds), Thinking. Readings in cognitive science. Cambridge : CUP, 411-420.

Corminboeuf, G. (2009). L'expression de l'hypothèse en français. Entre hypotaxe et parataxe. Bruxelles : De BoeckDuculot (coll. « Champs linguistiques »).

Corminboeuf, G. (2010). La causalité sans les connecteurs 'causaux'. Préalables épistémologiques. In Johnsen L. A., Corminboeuf G. \& Conti V. (éds), Entre syntaxe et discours, Linx, 62-63 (numéro double), 39-62.

Corminboeuf, G. (2013a). Négation et asyndète. In François J. \& al. (éds), La linguistique de la contradiction, Berne, P. Lang, 233-242.

Corminboeuf, G. (2013b). Une composante «émotive» dans les constructions articulées par un et d'opposition? Journal of French Language Studies, 23-3, 357-375.

Corminboeuf, G. (à paraître). L'effet d'attente déçue dans les constructions à valeur oppositive. In Sens attendu / inattendu dans les langues, Sekali M. \& Trévise A. (éds).

Cornulier de, B. (1985). Effets de sens. Paris : Minuit.

Deulofeu, H.-J. (1989). Les couplages de constructions verbales en français parlé : effet de cohésion discursive ou syntaxe de l'énoncé. Recherches sur le français parlé, 9, 111-141.

Ducrot, O. (1984). Le dire et le dit. Paris : Minuit.

García Negroni, M. (2003). Gradualité et réinterprétation. Paris : L’Harmattan.

Kitis E. (2000). Connectives and frame theory. The case of antinomial hypotextual and. Pragmatics \& Cognition, 82, 357-409.

Lakoff, R. (1971). If 's, and's, and but's about Conjunction. In Fillmore, C. \& Langendoen, D. (eds.), Studies in Linguistics Semantics. Holt, Reinhart and Wilson: New York, 115-150.

Le Bidois G. \& R. (1967). Syntaxe du français moderne : ses fondements historiques et psychologiques. 2 vol., Paris : A. et J. Picard

Mann, W. \& Thompson, S. (1985). Assertions from Discourse Structure. Proceedings of the Eleventh Annual Meeting of the Berkeley Linguistics Society, 245-258.

Mann, W. \& Thompson, S. (1988). Rhetorical Structure Theory: Toward a functional theory of text organisation. Text, 8-3, 243-281.

Marcu D. \& Echihabi A. (2002). An Unsupervised Approach to Recognizing Discourse Relations. Proceedings of the 40th Annual Meeting of the Association for Computational Linguistics (ACL-2002). Philadelphia, PA, July 7-12. 
Nølke H. (2001). La ScaPoLine 2001 : Version révisée de la théorie Scandinave de la Polyphonie Linguistique, Polyphonie linguistique et littéraire, 3, 43-65. En ligne: http://akira.ruc.dk/ Michel/Publications/nummer-3hel.pdf

Riegel M., Pellat J.-C. \& Rioul R. (1994). Grammaire méthodique du français. Paris : PUF.

Sekali, M. (2010). Coordination et dynamique discursive : étude comparative des coordonnants anglais and, or, but et for. In Florea L. \& al. (éds), Directions actuelles en linguistique du texte. Casa Cărţii de Ştiinţă : Cluj-Napoca, 235-245.

Spenader, J. \& Stulp, G. (2007). Antonymy and Contrast Relations. Seventh International Workshop on Computational Semantics. Tilburg. En ligne : http://www.ai.rug.nl/ spenader/public_docs/Spenader_Stulp.pdf

Spenader, J. \& Lobanova A. (2009). Reliable Discourse Markers for Contrast Relations. Eighth International Workshop on Computational Semantics. Tilburg. En ligne: http://www.aclweb.org/ anthology-new/W/W09/ W09-3719.pdf

Spenader J. \& Maier E. (2009). Contrast as denial in multi-dimensional semantics. Journal of pragmatics, 41, 17071726.

Taboada, M. (2006). Discourse Markers as Signals (or Not) of Rhetorical Relations. Journal of Pragmatics, 38-4, 567-592.

Taboada, M. (2009). Implicit and Explicit Coherence Relations. In Renkema J. (ed.), Discourse, of Course. Amsterdam/Philadelphia: John Benjamins, 127-140.

Txurruka, I. G. (2003). The Natural Language Conjunction and. Linguistics and Philosophy, 26-3, 255-285.

\section{Corpus oraux exploités}

[ofrom] Avanzi M. \& Béguelin M.-J. (2012). Présentation du corpus OFROM - corpus oral de français de Suisse romande. Université de Neuchâtel. http://www.unine.ch/ofrom.

[cid] Bertrand, R., Blache, P., Espesser, R., Ferré, G., Meunier, C., Priego-Valverde, B., Rauzy, S. (2008). Le CID Corpus of Interactional Data - Annotation et Exploitation Multimodale de Parole Conversationnelle. Traitement Automatique des Langues, 49-3.

[ctfp] Blanche-Benveniste, C., Rouget C. \& Sabio F. (éds) (2002). Choix de textes de français parlé : trente-six extraits. Paris : Champion.

[cfpp2000] Branca-Rosoff, S., Fleury, S., Lefeuvre, F. \& Pires, M. Discours sur la ville. Corpus de Français Parlé Parisien des années 2000 (CFPP2000). http://ed268.univ-paris3.fr/CFPP2000/

[crfp] Delic. (2004). Présentation du 'Corpus de Référence du Français Parlé'. Recherches sur le français parlé, 18, $11-42$.

[pfc] Durand, J., Laks, B. \& Lyche, C. (2002). La phonologie du français contemporain: usages, variétés et structure. In Pusch, C. \& Raible, W. (éds). Romanistische Korpuslinguistik - Korpora und gesprochene Sprache / Romance Corpus Linguistics - Corpora and Spoken Language. Tübingen : Gunter Narr Verlag, 93-106.

[pfc] Laks, B., Durand, J., Lyche, C. (2005). PFC : Un corpus numérisé pour la phonologie du français. In Williams, G. (éd.), Les linguistiques de corpus. Rennes : PUR, 205-217.

\section{Conventions de transcription des extraits de français parlé}

Les transcriptions des exemples oraux ont été revues par mes soins et ponctuées minimalement.

'+' signale une pause ou une fin d'énoncé.

':' marque un allongement.

'[]' signale un chevauchement.

'(XXX)' signale une séquence incompréhensible.

Les petites majuscules marquent un accent d'intensité.

Pour des raisons d'intelligibilité des exemples, j'ai ajouté des '?' pour les questions. 
${ }^{1}$ Le procédé est ancien. Antoine (1958: 886) souligne que « l'usage du et (nec) adversatif existait déjà en latin (...) : Omnia habeo, neque quicquam habeo (Térence) ». L'auteur cite également des exemples en ancien français, dont ces deux vers du XIVème siècle : Or sont ilz deables, et angles furent. (...) En hault furent, et or sont bas (Renart le contrefait).

${ }^{2}$ A l'exception notable cependant de la relation Explication de la SDRT (Txurruka 2003) et de certaines temporelles « greffées » (Deulofeu 1989) comme :

j'ai perdu mon père j'avais dix-huit ans (oral, cfpp2000, 07-01)

puis on est arrivé euh en Italie c'était huit heures du matin à peu près (oral, pfc, Roanne, 42acd1, lg)

${ }^{3}$ Les constructions adversatives qui présenteraient des énonciations nominales ou une forme non finie du verbe ne sont pas abordées dans le cadre de cette contribution.

${ }^{4}$ La relation Otherwise serait marquée en français par sinon ou autrement. Pour la relation Antithesis, les auteurs donnent les deux exemples illustratifs suivants :

(...) [this] illustrates a lack of jobs, not laziness. (cité par Mann \& Thompson 1988)

Players want the referee to balance a bad call benefiting one team with a bad call benefiting the other. As a referee, I just want to call each play as I see it. (cité par Mann \& Thompson 1985)

${ }^{5}$ On verra infra que Lakoff (1971) distingue deux relations contrastives. Dans la tradition de la RST, Spenader \& Lobanova (2009) en distinguent trois (Contrast, Antithesis et Concession).

${ }^{6}$ On voit que les exemples de Busquets cités supra étaient directement inspirés de ceux de Lakoff.

${ }^{7}$ Evidemment, cela n'empêche pas que l'on puisse mobiliser des processus inférentiels. Dans un contexte de visite guidée, si je dis en désignant les guides: Pierre parle le français, Jean parle l'allemand, l'inférence que l'on construit est que la visite de Pierre se déroulera en français et celle de Jean en allemand. Ou que Jean ne parle pas le français et Pierre ne parle pas l'allemand. Mais, quelle que soit l'inférence, elle n'exploite pas une relation de cause à effet entre les connaissances linguistiques de l'un et de l'autre des protagonistes.

${ }^{8}$ Un topos (ou garant) peut se définir comme un lieu commun informulé, mais consensuel dans une communauté linguistique. Les topoï légitiment un parcours argumentatif en faisant exister une règle d'inférence (présentée comme) fondée antérieurement. En disant il pleut donc je reste chez moi, je convoque le topos selon lequel «moins il fait beau, moins on est susceptible de sortir de chez soi ».

${ }^{9}$ On peut évidemment jouer du caractère sous-informatif d'un énoncé pour transmettre des contenus implicites. C'est le cas en particulier des tautologies et des questions rhétoriques.

${ }^{10}$ Les conventions de transcription pour les exemples oraux sont listées en fin de document, après la bibliographie.

${ }^{11}$ « Enchaînements marqués », parce qu'il faut souligner que les modèles disponibles dans la littérature scientifique sont destinés essentiellement à la description des enchainements marqués. Le non marquage est parfois intégré à la modélisation, mais il est ramené aux cas marqués sans qu'il y ait des incidences théoriques notables. Cet état de fait entraîne des conséquences malheureuses quant à la saisie de la spécificité du non marquage par rapport au marquage (supposé) explicite des relations de discours (Corminboeuf 2010).

${ }^{12}$ Le connecteur mais fonctionne dans des enchaînements très différents et est de plus compatible avec pourtant. Opposer les « enchaînements en pourtant » et les « enchaînement en mais » est un raccourci commode, mais qui ne laisse pas d'être caricatural.

${ }^{13}$ Le connecteur pourtant est peu optimal parce que l'énonciation c'est cassé ne constitue pas la révocation d'une attente forte projetée par David en a eu une.

${ }^{14}$ Cette question de l'opposition fonctionnelle entre solution asyndétique vs syndétique mériterait une étude en soi, où il conviendrait de distinguer la présence ou l'absence de et de la possibilité ou de l'impossibilité de son insertion, ce qui n'est pas la même chose.

${ }^{15}$ Le placement du fait étonnant en tête est très rare (environ 2,5\% des exemples). En voici une occurrence :

L'entraîneur du Bayern Munich, Louis Van Gaal, a qualifié de «folie» les tests physiques organisés la semaine prochaine par Joachim Löw en vue du Mondial 2010. (...) «On aurait pu donner nous-mêmes les résultats de nos tests physiques. Je n'ai jamais vécu quelque chose de similaire et j'ai déjà entraîné quelques 
grands clubs », a poursuivi le technicien néerlandais qui a notamment dirigé le FC Barcelone, l'Ajax Amsterdam et la sélection néerlandaise. (presse écrite)

On peut difficilement insérer contre toute attente et dire Je n'ai jamais vécu quelque chose de similaire et, contre toute attente, j'ai déjà entraîné quelques grands clubs, ce qui montre qu'il ne s'agit pas ici du déni d'une attente forte. Si ces enchaînements sont symétriques pour le linguiste (cf. ex. 31), ils ne le sont pas pour les sujets parlants.

${ }^{16}$ La lecture conséquentielle serait paradoxale, au sens de Carel \& Ducrot (1999). On pourrait considérer que l'enchaînement doxal constitue un guide pour l'interprétation (en plus du fait que la relation est souvent préfabriquée). Un enchaînement paradoxal serait dès lors peu préférentiel. Ainsi, le topos de l'escargot (il pleut donc je sors) est paradoxal: pas étonnant qu'il prête à sourire. Cependant, dans (35), il est difficile de savoir si l'enchaînement est doxal ou non, puisque cela dépend du topos que l'on mobilise.

${ }^{17}$ Sur environ 150 occurrences, dont un tiers d'exemples oraux, le corpus ne comporte que 3 exemples asyndétiques.

${ }^{18}$ De plus, certains emplois de mais en français n'ont rien de contrastif (il est bête mais bête! cf. Garcia Negroni 2003 ; voir aussi des énoncés de l'anglais comme he had but one friend). Si on inclut ces emplois, la relation Contraste court le risque d'être trop accueillante pour être consistante scientifiquement. Spenader \& Lobanova (2009) font la même observation pour le connecteur however - tenu pour un marqueur contrastif prototypique dans la littérature scientifique - et qui est en fait tout autant utilisé dans les relations de cause à effet que dans les contrastes.

19 Pour Sekali (2010: 236), and marquerait au moins 8 relations en anglais (accumulation, intensification, successivité temporelle, causalité, intention, concomitance, contraste, conséquence). 